GU J Sci, Part C, 6(2): 462-469 (2018)

Gazi Üniversitesi
Fen Bilimleri Dergisi
PART C: TASARIM VE TEKNOLOJI
dergipark.gov.tr/http-gujsc-gazi-edu-tr

\title{
Silah Sesleri Kullanılarak Ateșli Silahların Sınıflandırılmasında Akustik Parametrelerin Etkisi
}

\author{
Turgut ÖZSEVEN ${ }^{1, *}$, Muharrem DÜĞENCi' \\ ${ }^{\text {I} G a z i o s m a n p a s ̧ a ~ U ̈ n i v e r s i t e s i, ~ M u ̈ h e n d i s l i k ~ v e ~ D o g ̆ a ~ B i l i m l e r i ~ F a k u ̈ l t e s i, ~ B i l g i s a y a r ~ M u ̈ h e n d i s l i g ̆ i ~ B o ̈ l u ̈ m u ̈, ~ 60250, ~ T O K A T ~}$ \\ ${ }^{2}$ Karabük Üniversitesi, Mühendislik Fakültesi, Endüstri Mühendisliği Bölümü, 78050, KARABÜK
}

\begin{abstract}
$\ddot{O ̈ z}$
Makale Bilgisi

Başvuru: 26/12/2017

Düzeltme: 13/03/2018

Kabul:20/03/2018

Anahtar Kelimeler

Silah sesi tanıma Silah türü sinıflandırma

Akustik analiz özellikle malzeme bilimi ve insan seslerinde kullanılmakta olup son yıllarda savunma sanayiinde ses perdesi, silah ve nişancı tespiti, insansız hava araçları gibi bir çok alanda kullanılmaktadır. Ateşli silahlarla ilgili eylemler hem güvenlik güçleri hem de halk için artan bir endişe kaynağıdır. Silah seslerinin sınıflandırılması için ticari veya deneysel çeşitli çalışmalar mevcuttur. Bu çalışmanın amacı silah seslerini kullanarak ateşli silahların türünü tespit etmede akustik analizin etkilerinin incelenmesidir. Çalışmada 23 ateşli silaha ait 510 atış ses kaydı kullanılmıştır. Akustik analiz için formant frekansları, MFCC, LPCC ve enerji parametreleri incelenmiştir. Akustik parametrelerin silahları sınıflandırmadaki etkinliği istatistiksel olarak analiz edilmiş ve kullanılan tüm parametrelerin etkili olduğu görülmüştür. MLP sınıflandırıcı ile sınıflandırma performansı test edilmiş ve ateşli silah tanıma oranı \%71.56 elde edilmiştir. Tanıma oranı en yüksek silah türü "Carl Gustav M45" ve "Tokarev PPSh", tanıma oranı en düşük olan silah "Tikka Model T2" olarak tespit edilmiştir.
\end{abstract} Akustik analiz

Keywords

Gunshot recognition Firearm Classification Acoustic analysis

\section{The Impact of Acoustic Parameters on the Classification of Firearms Using Gunshots}

\begin{abstract}
Acoustic analysis is mainly used in material science and human voice. In recent years, many areas such as sound perception, gun and shooter detection, unmanned aerial vehicles have been used in the defense industry. Actions related to firearms are an increasing source of concern for both security forces and the public. There are various commercial or experimental studies for the classification of gunshots. The purpose of this study is to examine the effects of acoustic analysis on the detection of firearms by using gunshots. In the study, 511 gunshots recordings of 23 firearms were used. The formant frequencies, MFCC, LPCC and energy parameters for acoustic analysis are examined. The effectiveness of the acoustic parameters in classifying the weapons was analyzed statistically and all the parameters used were found to be effective. The classification performance was tested with the MLP classifier and the firearm recognition rate was $71.56 \%$. The "Carl Gustav M45-b" and "Tokarev PPSh" was identified as the weapon with the highest recognition rate and the lowest "Tikka Model T3" recognition rate.
\end{abstract}

\section{GÍRIŞ (INTRODUCTION)}

Silah seslerinin tespiti, sınıflandırması ve lokalizasyonu özellikle halk sağlığı, gözetim, kolluk kuvvetleri ve orduyla ilgili alanlarda önemlidir [1]. Silah sesleri için tasarlanmış çeşitli ticari ve deneysel sistemler mevcuttur. Bu sistemler ateşli silah türlerini sınıflandırma veya keskin nişancı yerini tespit etmeyi amaçlamaktadır [2]. Atış yerlerinin tespiti için ses özelliklerinin zemin, bina ve diğer yakın cisimlerden gelen ses yansıması kavramlarının bilinmesi gerekir [3].

Bir silah sesinin özellikleri mermi ve namlu kalibresi, ateşleme için kullanılan gazın kimyasal özellikleri gibi faktörlere bağlıdır. Ayrıca, silah sesleri mükemmel bir impulse sinyali olduğu için sıcaklık, rüzgar hızı ve hava nemi gibi faktörler hakkında bilgi sağlamaktadır $[1,3,4]$. 
Silah sesi tespitinde namlu ucu patlaması (muzzle blast) ve süpersonik mermi kavramları ön plana çıkmaktadır. Namlu ucu patlaması, merminin silah ucundan firlatılması için kullanılan patlama sonucunda namlu doğrultusunda oluşan ses dalgalarıdır. Bu ses dalgasının süresi 3-5 ms arasındadır. Akustik dalga olarak adlandırılan bu ses dalgası ses hızına sahiptir. Silah seslerinin kaydı yapılırken, kayıt cihazı silaha yakın ise temel akustik sinyal namlu ucu patlamasıdır. Eğer kayıt cihazı silaha yakın değilse yankılanmalar oluşacaktır. Namlu ucu patlaması sonrası mermi süpersonik bir hıza sahipse mermi yolundan dişarıya doğru akustik şok dalgası oluşturur [5-6].

Akustik, ses ile ilgilenen fizik dalıdır. Algısal değerlendirmenin kişiden kişiye değişiklik göstermesi nedeniyle objektif ses değerlendirme yöntemleri kullanılmaktadır [7]. Akustik analiz, ortam bağımsız olarak seslerin nesnel olarak değerlendirilmesi için kullanılan yöntemlerden birisidir [8]. Akustik analiz objektif, noninvaziv, kısa sürede veri sağlayan ve pahalı olmayan bir yöntem olup analiz için kullanılan ücretsiz yazılımlarda bulunmaktadır [9]. Temel olarak, akustik analiz ses kayıtlarının sayısal sinyal işleme yöntemleri ile işlenmesi sonucu sese ait çeşitli parametrelerin elde edilmesidir.

Mevcut durumda silah sesi ve atış yeri tespit etmek için birçok ticari sistem mevcuttur. Ancak, akustik öznitelikler yardımıyla gerçekleştirilen çalışmalar güncelliğini korumakta ve araştırmalar devam etmektedir. Silah sesleri ile yapılan çalışmalar esasında sinyal işleme yöntemlerini kapsamakta olup gömülü sistemler yardımıyla veya yazılımsal olarak gerçekleştirilebilir.

Askeri alanda kullanılan silah sesi algılama ve tanıma sistemleri, yaklaşı \%100 tanıma oranına, kısa tepki süresine $(<1 \mathrm{sn})$ ve düşük yanlış alarm oranına sahiptir. Bu sistemler, namlu (muzzle) patlaması ve/veya süpersonik mermi tarafindan üretilen akustik şok tespiti için geliştirilmiştir. Böyle bir sistemin tipik örneği, Boomerang [10] ya da onun öncülü Bullet Ears'tır. Her iki sistem de silah seslerini algılama ve lokalizasyon için mikrofon dizisi kullanır [11]. Shotspotter, şehirdeki sesleri dinleyerek silah sesi algıladığında güvenlik birimine haber verir [12]. ABD'nin birçok şehrinde kullanılmaktadır. Pinpoint [13] ve SWATS [14] ise keskin nişancı ve konumunu tespit etmek için askerlerin omuzlarına takılan sensörleri kullanır. Ancak, bu uygulamalar sadece silah sesi patlamasını tespit etmekte ve silah türü hakkında bilgi vermemektedir.

Silah sesleri yardımıyla silahların sınıflandırılması ve yer tespiti için fourier ve dalgacık dönüşümü, Saklı Markov Modeli (HMM - Hidden Markov Model), Gauss Karışım (GMM - Gaussian Mixtures Model) ve Ençok Olabilirlik (Maximum Likelihood) modelleri gibi birçok yöntem literatürde kullanılmıştır [1].

Yapılan çalışmaların çoğunluğu minimum gürültülü yüksek ses kalitesine sahip kayıtlar ile gerçekleştirilmiş ve \% $00-\% 100$ başarı oranına sahiptir.

Gerosa vd., silah sesi tespiti için kullanılabilecek en iyi öznitelik kümesini tespit etmek için 47 öznitelik içinden nasıl seçim yapılacağını incelemiştir. Silah sesi ile gürültüyü ve çığlık ile gürültüyü birbirinden ayırt edebilmek için paralel çalışan iki GMM, ses kaydı olarak da 10 atış ve 12 alkış sesi kullanılmıştır. Yüksek gürültülü ortamda \%90'lık başarı elde edilmiştir [15].

Freire ve Apolinário gürültülü ortamlarda 8 Doğrusal Öngörü Kepstral Katsayıları (LPCC - Linear Predictive Cepstral Coefficient) katsayıs1, 13 Mel-Frekans Kepstral Katsayıları (MFCC - Mel Frequency Cepstral Coefficients) katsayısı, korelasyon ve dürtüsellik yöntemlerini karşılaştırmıştır ve dört kategoride (ateşli silah, balon, konuşma, alkış) 88 ses sınıflandırılmaya çalışılmıştır. HMM sınıflandırıcı kullanılmıştır. Dürtüsellik ölçümü birkaç yanlış sınıflandırma ile sonuçlanırken, MFCC ve Lineer Kestirimci Kodlama (LPC - Linear Predictive Coding) gürültüsüz orijinal sinyaller için \% 100 algılama oranına ulaşmıştır. Yaklaşım daha sonra $30 \mathrm{~dB}, 25 \mathrm{~dB}$ ve $20 \mathrm{~dB}$ sinyal-gürültü oranı (SNR - Signal to Noise Ratio) ile karşılaştıııldığında LPC ve MFCC düşük tanıma oranlarıyla (sırasıyla\% 59 ve\% 31) ve SNR = 30dB'de dahi yüksek yanlış alarm oranlarıyla bitiyor. 20dB SNR için \%79.8 başarı elde edilmiştir [16].

Çoklu dalgacık dönüşümü ve medyan filtreleme gibi birçok yöntemin karş1laştırıldığı çalışmada SNR dikkate alınmamıştır [1]. Silah sesi sınıflandırmak için MFCC ile LPC öznitelikleri, çaprazkorelasyon ve SVM sınıflandırıcı kullanılan çalışmada, farklı atış mesafelerinden G3 ve MP5 silahlar ile elde edilen 332 atış ve 102 yabancı ses kaydı kullanılmış, LPC ve çaprazkorelasyon ile Destek Vektör Makineleri (SVM - Support Vektor Machine) sınıflandırıcıda \%99.7’lik bir doğru sınıflandırmaya ulaşılmıştır [2]. 
17 farklı silaha ait 5 silah sınıfı kullanılan çalışmada LPC, Algısal Doğrusal Tahmin (PLP - Perceptual Linear Prediction), Bağıl Spektral Dönüşüm PLP (Rasta PLP - Relative Spectral Transform PLP) ve MFCC öznitelikleri hiyerarşik sınıflandırıcı ile sınıflandırılmış \%96.29 başarı elde edilmiştir [17].

Suman vd., 150 silah sesi ile MFCC ve üç katmanlı Yapay Sinir Ağları (YSA) sınıflandırıcı ile gürültüsüz ortamda $\% 95$ ve gürültülü ortamda $\% 85$ başarı elde etmiştir [18].

Normal hava koşullarında 4 farklı silahtan atılan silah sesleri üzerinden elde edilen MFCC, sıfır geçiş sıklığı, izgel yüzde, izgel merkez ve genişlik, izgel düzlük ölçütü öznitelikleri kullanılmıştır. En yüksek başarı k-en yakın komşu (k-NN - k nearest neighbohood) ile \%80 olarak tespit edilmiştir [5].

EAR-TUKE olarak akustik olay tespit sistemi sunulan çalışmada HMM ve MFCC, FBANK ve MELSPEC kullanılarak cam kırılması ve silah sesini \%98.89 başarı ile ayırt etmişti. 46 silah sesi ve 13 cam kırılması sesi kullanılmıştır [19].

Yapılan çalışmalardan elde edilen sonuçlar silah türünün belirlenmesinde ve uzaktan algılanmasında akustik analizin kullanılabilecek bir yöntem olduğunu göstermekle birlikte güncelliğini koruyan bir çalışma alanıdır. Bu çalışmanın amacı silah seslerini kullanarak ateşli silahların türünü tespit etmede akustik analizin etkilerinin araştırılmasıdır. Çalışmanın 2. bölümünde kullanılan materyaller ve yöntemler, 3. Bölümde ise elde edilen sonuçlar verilmiştir.

\section{MATERYAL VE METOD (MATERIAL AND METHOD)}

\subsection{Kullanılan Ses Kayıtları (The Used Voice Recordings)}

Çalışmada Airborne Sound'a ait Still North Media [20] tarafindan oluşturulan ateşli silah ses kayıtları kullanılmıştır [21]. Ses kayıtları 44.1 kHZ, 16-bit, 320 kb MP3 formatındadır. Kullanılan ses kayıtlarına ait dağılım Tablo 1'de ve ses kayıtlarından bir örnek Şekil 1'de verilmiştir.

Tablo 1. Çalışmada kullanılan ses kayttlarına ait özet bilgi (Summary information about voice recordings used in the study)

\begin{tabular}{|c|c|c|c|}
\hline S.No & Kategori & Silah & Ses Kaydı Sayısı \\
\hline 1 & \multirow{5}{*}{ Pompalı Tüfek } & Winchester Model 1894 & 19 \\
\hline 2 & & Mossberg Model 190 & 20 \\
\hline 3 & & Benelli Nova 12 & 18 \\
\hline 4 & & Charles Daly CD FTAW 12 & 18 \\
\hline 5 & & Winchester Model 1912 & 21 \\
\hline 6 & \multirow{9}{*}{ Tüfek } & Arisaka & 49 \\
\hline 7 & & Springfield 1917 & 8 \\
\hline 8 & & Tikka Model T3 & 17 \\
\hline 9 & & Savage Model 10 & 31 \\
\hline 10 & & Mosin Nagant & 19 \\
\hline 11 & & Marlin 336 & 16 \\
\hline 12 & & Ruger $10 / 22$ & 20 \\
\hline 13 & & Norinco SKS & 20 \\
\hline 14 & & AR-15 M4 .223 & 27 \\
\hline 15 & \multirow{3}{*}{ Makinalı Tüfek } & AK-47 & 53 \\
\hline 16 & & Tokarev PPSh & 34 \\
\hline 17 & & Carl Gustav M45 & 25 \\
\hline 18 & \multirow{4}{*}{ Tabanca } & Ruger Mark III & 10 \\
\hline 19 & & Bersa .380 & 13 \\
\hline 20 & & 1911.45 & 12 \\
\hline 21 & & Walther PPQ & 24 \\
\hline 22 & \multirow{2}{*}{ Toplu Tabanca } & Smith \& Wesson 642 & 21 \\
\hline 23 & & Ruger Single Six & 15 \\
\hline & & TOPLAM & 510 \\
\hline
\end{tabular}




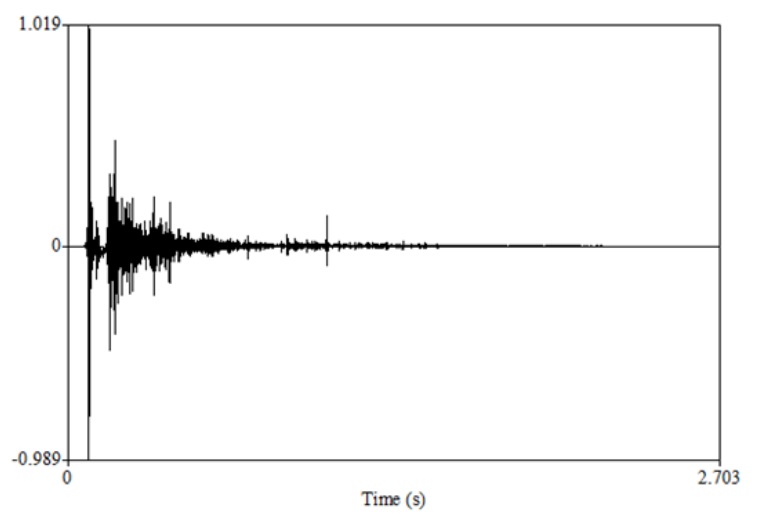

Şekil 1. Mossberg Model 190 için örnek bir ses kaydl (Sample voice recording for Mossberg Model 190)

\section{2. Önişleme Yöntemi (Pre-processing Method)}

Ses kayıtları stereo olduğu için öznitelik çıkartımı öncesi stere-mono dönüşümü ile monoya dönüştürülmüştür. Stereo-mono dönüşümü için Eşitlik 1'de verilen formül kullanılmıştır.

$X_{\text {mono }}=\frac{X_{\text {stereo }}(1)+X_{\text {stereo }}(2)}{\max \left(\left|X_{\text {stereo }}(1)+X_{\text {stereo }}(2)\right|\right)}$

Akustik analiz ile akustik parametreler elde edilirken ses sinyalinin tamamı ayı anda değil sinyalden küçük kesitler alınır. Bunun nedeni, ses sinyallerinin küçük zaman aralıklarında kararlı kalmasıdır. Ses sinyallerinden bu şekilde küçük kesitler alma işlemi çerçeveleme olarak adlandırılır. Çerçeveler arasındaki geçişi yumuşatmak ve bilgi kaybını önlemek için bir sonraki çerçeve ile bir önceki çerçeve belirli oranda kesiştirilir. Bu işlem örtüşme oranı olarak adlandırılır [22]. Çerçeve boyutu $20 \mathrm{~ms}$ ve örtüşme oranı $\% 50$ belirlenmiştir. Sinyaldeki spektral sızmayı ve örtüşmeden dolayı oluşan kesişimi düzenlemek amacıyla pencereleme kullanılmaktadır [23, 24]. Ses sinyalleri işlenirken fourier dönüşümü sonrası spektral sızmayı önlemek için literatürde yoğun olarak kullanılan Hamming pencereleme yöntemi kullanılmıştır [24].

\subsection{Akustik Parametreler (Acoustic Parameters)}

Akustik analiz, sesten çeşitli parametreler elde edilerek seslerin nesnel olarak değerlendirmesi için kullanılır. $\mathrm{Bu}$ amaçla kullanılabilecek hazır yazılımlar mevcuttur [9]. Akustik analiz ve akustik parametrelerin tespiti için Hidden Markov Toolkit [25], COLEA [26], Praat [27] ve OpenSMILE [28] yazılımları birçok çalışmada kullanılmıştır. Grafiksel arayüz, toplu dosya işleme, ön işleme ve kullanım kolaylığı açısından incelendiğinde Praat yazılımı ön plana çıkmaktadır. Bu çalışmada her bir ses kaydı üzerinden akustik parametrelerin tespiti için Praat kullanılmış ve Tablo 2'de verilen parametreler elde edilmiştir.

Tablo 2. Kullanulan akustik parametreler (The used acoustic parameters)

\begin{tabular}{|l|l|c|}
\hline Akustik Parametre & İstatistiksel Varyasyonları & $\begin{array}{c}\text { Öznitelik } \\
\text { Sayısı }\end{array}$ \\
\hline Formant Frekansları (F1,F2,F3) & $\begin{array}{l}\text { Ortalama, min, maks, aralık, ortanca, standart } \\
\text { sapma }\end{array}$ & 18 \\
\hline $\begin{array}{l}\text { Formant Frekansları (F1,F2,F3) Bant } \\
\text { Genişliği }\end{array}$ & Ortanca & 3 \\
\hline 13 MFCC & Ortalama, standart sapma, ortanca & 39 \\
\hline 13 LPCC & Ortalama, standart sapma, ortanca & 39 \\
\hline & \multicolumn{1}{c}{ TOPLAM } & 99 \\
\hline
\end{tabular}


Formant sesin oluşumu esnasında oluşan rezonanstır ve sesin oluşumu ile ilgili spektral bilgi sağlamaktadır. Teoride çok sayıda formant vardır. Ancak, yalnızca ilk 3 veya 4 formant önemli bilgiler içerir [29]. Sesteki değişimin oluşum şekli formant bant genişliklerinde farklılıklara neden olur. MFCC, mel ölçeği aracılığı insan işitme sistemini modellemektedir. Üçgen bir dizi filtre aracılığı MFCC parametreleri elde edilmektedir [30]. Mel ölçeği, algılanan perde seviyesinin bir ölçüsüdür ve algılanan frekans ile ölçülen frekansı ilişkilendirir.

Sesler analiz edilirken çerçeveleme kullanıldığı için bir ses kaydı üzerinde birden fazla çerçeve yer almaktadır. Akustik parametreler her çerçeve için ayrı ayrı elde edilmektedir. 5 çerçeve içeren bir ses kaydı için F1 değerinden 5 adet yer alacaktır. İstatistiksel yöntemlerle (ortalama, standart sapma vb.) 5 özellik 1'e indirgenmektedir. Tablo 2'de yer alan istatistiksel varyasyonlar bu şekilde hesaplanmıştır.

\subsection{Sinıflandırıcı (Classifier)}

Silah seslerinin sınıflandırılması için Çok Katmanlı Algılayıcı (MLP - Multilayer Perceptron) kullanılmıştır. MLP, birden fazla gizli katmana sahip olan ve genellikle ileri yönlü (feed-forward) bağlantılı bir yapay sinir ağı modelidir. MLP en çok kullanılan YSA türüdür. Sınıflandırma işlemi WEKA [31] ile gerçekleştirilmiştir. Şekil 2'de çalışmada kullanılan MLP modeli verilmiştir.

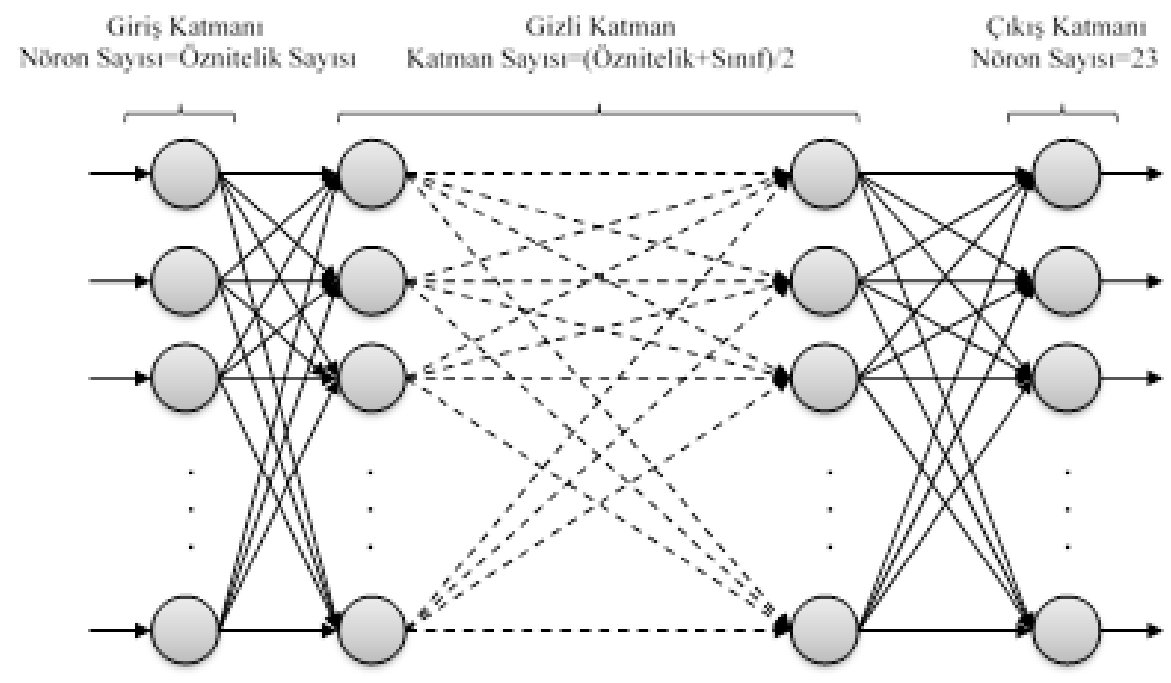

Şekil 2. Kullanılan MLP modeli (The used MLP model)

Giriş katmanında, öznitelikler ağa sunulur ve buradaki nöron sayısı öznitelik sayısı kadardır. Bu katman veriler üzerinde herhangi bir işlem yapmadan gizli katmana sunar. Gizli katman ağın temel işlevlerinin gerçekleştirildiği yerdir. Buradaki katman sayısı ve nöron sayısı MLP'nin kullanım amacina göre değişiklik göstermektedir. Gizli katmanda ağın kullandığı fonksiyon ile işlenen veri ağın çıkışını oluşturur. Çıkış katmanında kullanılan nöron sayısı ağa sunulan verinin çıkışı kadardır.

MLP modelinde öğrenme oranı 0.3 , momentum 0.2 ve epoch 500 kullanılmıştır. Gizli katman sayısı (öznitelik+sınıf sayısı)/2 olarak belirlenmiştir. Sınıflandırma sonuçlarının tutarlı olması ve sınıflandırma başarısını test etmek için veri kümesi 10 katlamalı çapraz doğrulama ile eğitim ve test olarak ayrılmıştır. Öznitelik kümelerinin etkinliğini araştırmak için sınıflandırma üç ayrı gruba ayrılan öznitelik kümeleri üzerinde her bir grup ve bu grupların birleşimi için ayrı ayrı gerçekleştirilmiştir.

\section{SONUÇLAR VE TARTIŞMA (CONCLUSION AND DISCUSSION)}

Çalı̧̧mada kullanılan 510 ses kaydı için formant frekansları, MFCC ve LPCC özniteliklerini içeren 99 öznitelik Praat ile elde edilmiş ve sınıflandırma işlemi WEKA üzerinde MLP ile gerçekleştirilmiştir. Sınıflandırma başarıları üç öznitelik kümesi için ayrı ayrı ve bunların birleşimi ile değerlendirilmiştir. Elde edilen sonuçlar Tablo 3'de verilmiştir. 
Tablo 3. Sinıflandırma sonuçları (Classification results)

\begin{tabular}{|l|c|c|c|}
\hline Akustik Parametre & Gizli Katman Sayısı & Öznitelik Sayısı & Başarı \\
\hline Formant Frekansları & 22 & 21 & $\% 43.14$ \\
\hline MFCC & 31 & 39 & $\% 71.56$ \\
\hline LPCC & 31 & 39 & $\% 25.29$ \\
\hline Formant Frekansları+MFCC & 42 & 60 & $\% 70.20$ \\
\hline Formant Frekansları+LPCC & 42 & 60 & $\% 46.27$ \\
\hline MFCC+LPCC & 50 & 78 & $\% 70.39$ \\
\hline Tümü & 61 & 99 & $\% 68.24$ \\
\hline
\end{tabular}

Sınıflandırma sonuçları incelendiğinde LPCC ve formant frekanslarının silah seslerini sınıflandırmada yetersiz kaldığı ve tüm öznitelik kümesine göre sadece MFCC kümesinin daha yüksek sınıflandırma başarısı elde ettiği tespit edilmiştir.

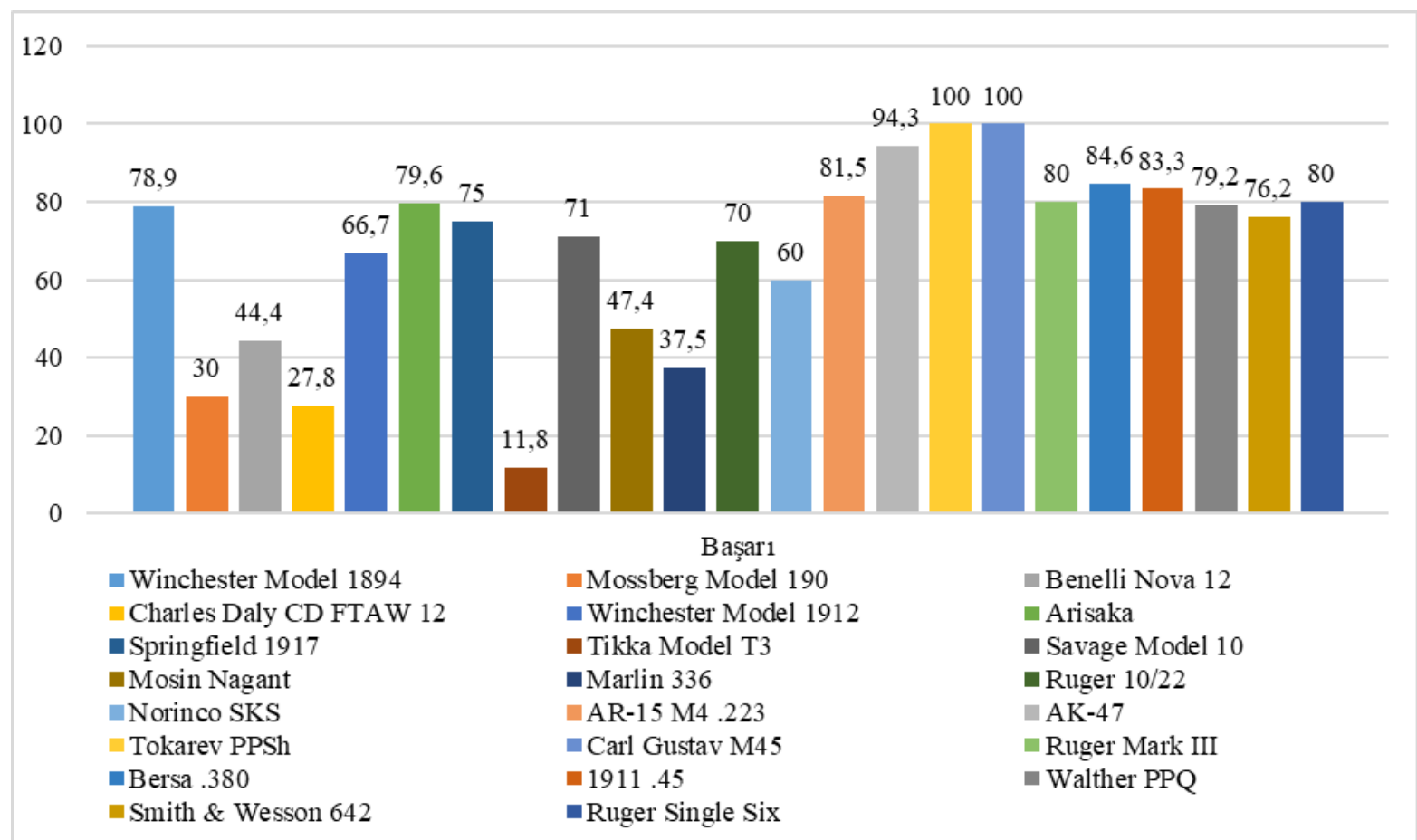

Şekil 3. MFCC öznitelik kümesinin sinıf bazlı başarı grafiği (Class-based success graph of the MFCC attribute set)

Şekil 3'e göre en yüksek tanıma oranına Carl Gustav M45 ve Tokarev PPSh sahipken en düşük tanıma oranına Tikka Model T3 sahiptir. Sonuç olarak akustik analiz silah seslerini silah türüne göre sınıflandırmada etkin olarak kullanılabilmiştir.

Aynı veri seti kullanılarak yapılan çalışma bulunmaması elde edilen sonuçların diğer çalışmalardan elde edilen sonuçlarla karşılaştırmasını kısıtlamaktadır. Ancak, elde edilen sonuçlar genel anlamda literatürle örtüşmekte ve silah türünün tespitinde akustik analiz ve silah seslerinin etkili bir şekilde kullanılabileceğini göstermektedir.

Elde edilen \% 71.56 sınıflandırma başarısı askeri uygulamalar için düşük bulunabilir. Bu çalışmada, ateşli silahların sınıflandırılmasında akustik analiz yöntemlerinin etkinliği araştırılmış olup. Ayrıca, öznitelik kümesi başarılar karşılaştırılmıştır. Bu nedenle, sınıflandırıcı performans karşılaştırması veya başarı oranını yükseltmeye yönelik incelemeler yapılmamıştır. Elde edilen başarı oranını artırmak için öznitelik seçim yöntemleri, farklı sınıflandırma metodları ve sınıflandırma öncesi ön işleme yöntemleri sürece dahil edilebilir.

Bu çalışmada gürültü faktörü göz önüne alınmamıştır. Ayrıca, bazı ses kayıtları tek atış bazı ses kayıtları çift atış sesi içermektedir. Bu durumların göz ardı edilmesi çalışmanın sınırlılığını oluşturmaktadır. 
Gelecek çalışmalar, bu sınırlılıklar göz önüne alınarak ve namlu ucu patlaması ile süpersonik mermi durumları göz önüne alınarak şekillendirilebilir. Ayrıca, sınıflandırma başarısını artırmaya yönelik çalışmalar yapılabilir.

\section{KAYNAKLAR (REFERENCES)}

[1] Chacon-Rodriguez, A., Julian, P., Castro, L., Alvarado, P. and Hernández, N., "Evaluation of Gunshot Detection Algorithms", IEEE Transactions on Circuits and Systems I: Regular Papers, Cilt 58, No 2, 363-373, 2011.

[2] Ahmed, T., Uppal, M. and Muhammad, A., "Improving Efficiency and Reliability of Gunshot Detection Systems", 2013 IEEE International Conference on Acoustics, Speech and Signal Processing (ICASSP), 513-517, May 2013.

[3] Maher, R. C., "Modeling and Signal Processing of Acoustic Gunshot Recordings", In Digital Signal Processing Workshop, 12th-Signal Processing Education Workshop, 257-261, September 2006.

[4] Fernández, A. I. T., "Propagación del Sonido En Bosques: Análisis Comparativo De Las Medidas in Situ", En Laboratorio y de Los Valores Predichos Por un Modelo, 2002.

[5] Arslan, Y. and Güldoğan, B., "Impulsive Sound Detection and Gunshot Recognition", In Signal Processing and Communications Applications Conference, 511-514, May 2015.

[6] Maher, R. C., "Acoustical Characterization of Gunshots", In Signal Processing Applications for Public Security and Forensics, 1-5, April 2007.

[7] Kılıç, M.A, ve Okur E., "CSL ve Dr.Speech ile Ölçülen Temel Frekans ve Pertürbasyon Değerlerinin Karşılaştırılması”, K.B.B. İhtisas Dergisi, No 8, 152-157, 2001.

[8] Özbal, E.A.,"Septum Devisyonlu Hastaların Septoplast Operasyonu Öncesi ve Sonrası Akustik Ses Analizi ile Değerlendirilmesi”, Uzmanlık Tezi, Şişli Etfal Hastanesi, İstanbul, 2008.

[9] Sataloff, R. T., "Treatment of Voice Disorders", Plural Publishing, 2005.

[10] İnternet: "Raytheon: Customer Success Is Our Mission", http://www.raytheon.com/, Son Erişim Tarihi: 20.02.2017.

[11] Hrabina, M. and Sigmund, M., "Acoustical Detection of Gunshots", 25th International Conference In Radioelektronika, 150-153, April 2015.

[12] İnternet: "ShotSpotter Gunshot Detection and Location Service", http://www.shotspotter.com, Son Erişim Tarihi: 20.02.2017.

[13] İnternet: "Cobham PLC”, http://www.cobham.com, Son Erişim Tarihi: 20.02.2017.

[14] İnternet: "QinetiQ North America”, https://www.qinetiq-na.com, Son Erişim Tarihi: 20.02.2017.

[15] Gerosa, L., Valenzise, G., Tagliasacchi, M., Antonacci, F. and Sarti, A., "Scream and Gunshot Detection in Noisy Environments”, In Signal Processing Conference, 1216-1220, September 2007.

[16] Freire, I. L. and Apolinário Jr, J. A., "Gunshot Detection in Noisy Environments", In Proceeding of the 7th International Telecommunications Symposium, Manaus, Brazil, 1-4, September 2010.

[17] Djeddou, M. and Touhami, T., "Classification and Modeling of Acoustic Gunshot Signatures", Arabian Journal for Science \& Engineering, Cilt 38, No 12, 2013.

[18] Suman, P., Karan, S., Singh, V. and Maringanti, R., "Algorithm for Gunshot Detection Using Melfrequency Cepstrum Coefficients (MFCC)", In Proceedings of Ninth International Conference on Wireless Communication and Sensor Networks, 155-166, 2014. 
[19] Lojka, M., Pleva, M., Kiktová, E., Juhár, J. and Čižmár, A., "Efficient Acoustic Detector of Gunshots and Glass Breaking”, Multimedia Tools and Applications, Cilt 75, No 17, 10441-10469, 2016.

[20] İnternet: "Still North Media", https://www.stillnorthmedia.com, Son Erişim Tarihi: 20.02.2017.

[21] İnternet: “Airborne Sound”, http://www.airbornesound.com, Son Erişim Tarihi: 20.02.2017.

[22] Rabiner, L. R. and Schafer, R. W., "Digital Processing of Speech Signals", Prentice Hall, (1978).

[23] Ertürk, S., "Sayısal İşaret İşleme", Birsen Yayınevi, İstanbul, (2002).

[24] Tarng, W., Chen, Y.-Y., Li, C.-L., Hsie, K.-R., and Chen, M., "Applications of support vector machines on smart phone systems for emotional speech recognition", World Academy of Science, Engineering And Technology, 72106-113 (2010).

[25] Bou-Ghazale, S. E. and Hansen, J. H., "A comparative study of traditional and newly proposed features for recognition of speech under stress", Speech And Audio Processing, IEEE Transactions On, 8 (4): 429-442 (2000).

[26] Loizou, P., "Colea: A MATLAB software-tool for Speech Analysis", University Of Arkansas, Arkansas, (2003)

[27] Boersma, P. P. G., "Praat, a System for Doing Phonetics by Computer", Glot international, No 5, 2002.

[28] Eyben, F., Wöllmer, M., and Schuller, B., "Opensmile: The munich versatile and fast open-source audio feature extractor", Proceedings of the 18th ACM International Conference on Multimedia, Frenze, 1459-1462 (2010).

[29] Rezaei, N. and Salehi, A., "An antroduction to speech sciences (acoustic analysis of speech)", Iranian Rehabilitation Journal, 4 (4): 5-14 (2006).

[30] Sethu, V., "Automatic emotion recognition: An investigation of acoustic and prosodic parameters", Doktora Tezi, The University of New South Wales Electrical Engineering \& Telecommunications, Sydney, (2009).

[31] Hall, M., Frank, E., Holmes, G., Pfahringer, B., Reutemann, P. and Witten, I. H., "The WEKA Data Mining Software: An update”, ACM SIGKDD Explorations Newsletter, Cilt 11, No 1, 10-18, 2009. 